\title{
TELECOMMUNICATION FREQUENCY BAND SPECTRUM OCCUPANCY IN KAMPALA UGANDA
}

\author{
Gertrude Ayugi $^{1}$, Akisophel Kisolo ${ }^{2}$, Tumps.W. Ireeta $^{3}$ \\ ${ }^{1}$ PhD student Department of Physics, Makerere University Kampala, Uganda. P.O Box 7062, Kampala \\ ${ }^{2}$ Senior Lecturer, Department of Physics, Makerere University Kampala, Uganda. P.O Box 7062, Kampala \\ ${ }^{3}$ Senior Lecturer, Department of Physics, Makerere University Kampala, Uganda. P.O Box 7062, Kampala \\ agertrude@cns.mak.ac.ug,akisolo@cns.mak.ac.ug, ireeta@cns.mak.ac.ug
}

\begin{abstract}
The Effective utilization of the radiofrequency electromagnetic spectrum is paramount for wireless communication systems especially in telecommunication. This paper presents measurements and analysis of spectrum occupancy in the telecommunication frequency bands performed in Kampala, Uganda in March 2015. The telecommunication bands considered in this study are GSM900, GSM1800, UMTS2100 and LTE2600. Occupancy measurements have been carried out in an outdoor setting using the Aaronia Hyperlog 70600 antenna and the Spectran HF-6065 V4.The analysis shows that the licensed telecommunication spectrum bands are not fully utilized. Highest spectrum occupancy of 52.4\% was observed in the GSM900 Downlink telecommunication band while the lowest occupancy of approximately $0 \%$ was observed in the LTE2600 band.
\end{abstract}

Key words: spectrum occupancy, wireless telecommunication, Duty cycle, Threshold

\section{INTRODUCTION}

The radiofrequency electromagnetic spectrum is a finite and scarce resource essential in wireless communication systems. Various wireless applications and services have developed with demand for wireless broadband common in telecommunications service providers. Most of the available radiofrequency spectrum has already been allocated and the limited spectrum resources has become a serious issue in Uganda.

However, some recent spectrum measurements have demonstrated that most of spectrum, though allocated, is vastly underutilized [1]. The traditional spectrum allocation policy used in Uganda, which provides a fixed allocation to the license user, is successful in reducing interference but does not offer flexible and efficient utilization of Radiofrequency spectrum.

\section{RELATED WORK}

Wireless spectrum occupancy measurement campaigns covering both a wide frequency range as well as some specific licensed bands have already been performed in a number of locations and scenarios [2, 3]. These measurements have been performed to determine the level to which allocated radiofrequency spectrum bands are occupied in wireless communication systems.

Spectrum occupancy investigation: Measurements were done in South Africa by Barnes. Occupancy in the UHF band was found to be approximately $20 \%$. The GSM900 $\mathrm{MHz}$ band had the highest occupancy of approximately $92 \%$ and GSM 1800 band had approximately 40\% [4]

Bara'u, carried out a study titled An Insight into Spectrum
Occupancy in Nigeria. This study was carried out in indoors within the region of $700 \mathrm{MHz}$ to $2.5 \mathrm{GHz}$ in Abuja, Nigeria. The results obtained indicate that large portion of the allocated spectrum is underutilized which could be considered for the deployment of cognitive radio paradigm in the near future.[5]

Jiantao_carried out Spectrum Occupancy Measurements and Analysis in Beijing in 2012. The frequency bandwidth considered in the study was $450-2700 \mathrm{MHz}$. The measurement results showed that the actual spectral occupancy in Beijing is about $13.5 \%$ [6].

An evaluation of spectrum occupancy in an urban environment in a cognitive radio context covering the frequency range from $25 \mathrm{MHz}$ up to $3.4 \mathrm{GHz}$ was done in Bucharest, Romania. The mean spectrum occupancy ratio over the whole band was as low as $12.19 \%$ [7].

In 2005, spectrum occupancy measurements were performed by Shared Spectrum Company in conjunction with the Wireless Interference $\mathrm{Lab}$ of the Illinois Institute of Technology in Chicago, Illinois, Measurements were made in all bands in the $30 \mathrm{MHz}$ to $3000 \mathrm{MHz}$ range. The overall average spectrum usage during the measurement period was $17.4 \%$ or less [8].

Most of the Spectrum occupancy measurements conducted [9-11] have proved that the spectrum utilization level is low. A spectrum occupancy measurement has been performed in the frequencies occupied by mobile telecommunication bands in Kampala capital city of Uganda. The telecommunication bands considered are GSM900, GSM1800, UMTS2100 and LTE2600 which are allocated the $880-960,1710-1880,1920-2170$ and $2500-2690 \mathrm{MHz}$ respectively. 


\section{MATERIALS AND METHOD}

\subsection{Location}

The measurements were conducted at the roof top of the Mandela Hall Uganda at Kyambogo University. This site is located at Latitude 0.346443, Longitude: 32.630746. It has a direct line-of-sight with various base stations and telecommunication systems and also in an urban setting with a presumed high level of wireless activity. This site being in direct line of site allowed measurement of signals which have not encountered any distortion in form of reflection, refraction or scattering. A google map of the measurement site location is shown in figure 1

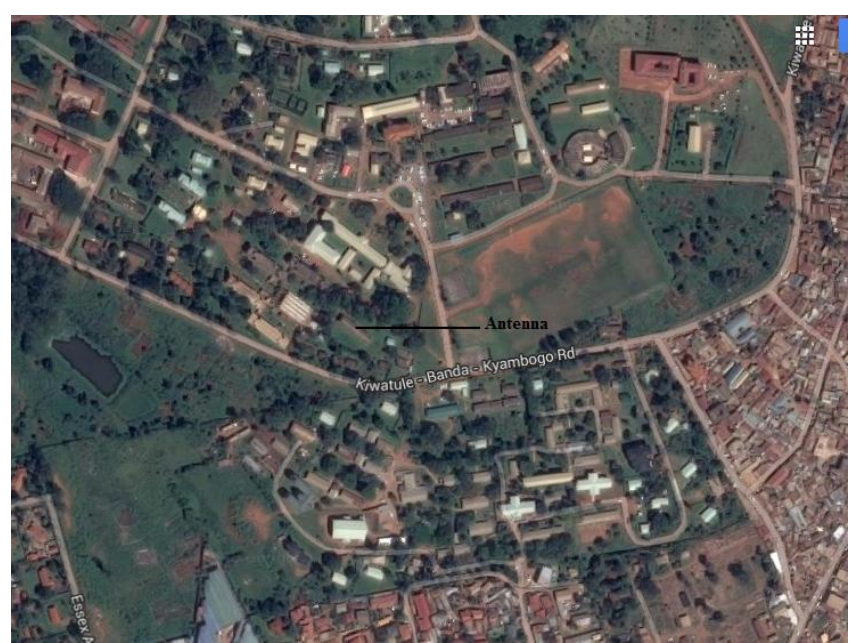

Figure 1: Measurement Location

\subsection{Measurement Setup}

The measurement setup used consists of a calibrated Aaronia Spectran HF-6065 V4 spectrum analyzer with a range of $10 \mathrm{MHz}-6 \mathrm{GHz}$, an Aaronia AG Hyperlog 70600 antenna with a range of $700 \mathrm{MHz}$ to $6.0 \mathrm{GHz}$, a laptop that is connected to the spectrum analyzer via a USB cable, and an MCS software specially designed to run on Aaronia spectrum analyzers. The MCS software is easily configured to suit a given measuring. The setup is connected as shown in figure 2. MATLAB software package was used to process and analyze the data to estimate the spectrum occupancy of the telecommunication bands.

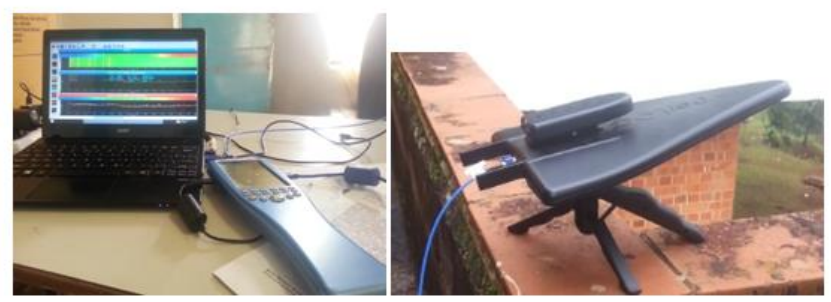

Figure 2 Spectran HF 6065 V4 interfaced to laptop and Antenna on top of the building

The full frequency range of the measurement study (700 $\mathrm{MHz}$ to $3000 \mathrm{MHz}$ ) was overviewed for 24 hours before analyzing to band by band telecommunication spectrum occupancy. Based on this overview, the active telecommunication bands were identified and their duty cycle/ occupancy measured. Each band was measured over a 24 hour interval. The configuration of the parameters for the spectrum analyzer during the measurements are given in Table I.

Table 1: Spectrum Analyzer Parameters configuration

\begin{tabular}{|l|l|}
\hline Parameter & Value \\
\hline Frequency Range & $700 \mathrm{MHz}-3000 \mathrm{MHz}$ \\
\hline $\begin{array}{l}\text { Telecommunication } \\
\text { frequency bands }\end{array}$ & $\begin{array}{l}\text { GSM900 } \\
\text { GSM1800 } \\
\text { UMTS2100 } \\
\text { LTE2600 }\end{array}$ \\
\hline Resolution Band width & $30 \mathrm{kHz}$ \\
\hline Video Band width & $30 \mathrm{kHz}$ \\
\hline Sweep time & $5 \mathrm{~ms}$ \\
\hline Detection type & RMS \\
\hline Sample points & 1000 \\
\hline Attenuation factor & Auto \\
\hline Reference level & -10 \\
\hline
\end{tabular}

\subsubsection{Duty Cycle (Measured Occupancy)}

Duty cycle indicates how often the signal is seen on each channel during a sample period [1]. The duty cycle can be obtained using:

$$
\text { Duty Cycle }=\frac{\text { Signal Occupation Period }}{\text { Total Observation Period }} \times 100 \%
$$

\subsubsection{Threshold Detection}

In spectrum occupancy measurements, determining the decision threshold upon which a particular channel can be deemed as free or busy is very important especially when energy detection is employed. In energy detection, no prior knowledge of the signal is known therefore it's very important to correctly determine the threshold for accurate readings. Setting the threshold metric too high will lead to under estimation of the spectrum while low decision metric will lead to over estimation of the spectrum. The noise floor can be obtained by replacing the antenna with a $50 \Omega$ resistor or similarly measure the noise floor by removing the antenna and not replacing it with anything for each measured band.

For each telecommunication band, the setup was left to run for one hour to obtain noise at each frequency. At each frequency, decision of threshold was fixed such that the false alarm probability was $1 \%$.

\section{RESULTS}

Figure 3 shows the $700-3000 \mathrm{MHz}$ spectrum. This spectrum shows that overall spectrum occupancy is very low, Analysis of this full band does not give an insight in analysis of the telecommunication bands utilization. The frequencies 
below $800 \mathrm{MHz}$ according to the Uganda communication commission are allocated to Digital Video Broadcasting Terrestrial (DVB-T) services. The peak at $900 \mathrm{MHz}$ is for GSM900, at $1800 \mathrm{MHz}$ is for GSM1800, at $2100 \mathrm{MHz}$ is for UMTS2100, at $2400 \mathrm{MHz}$ is for WLAN and the one at
$2600 \mathrm{MHz}$ is for LTE2600.

The red curve is for maximum power recorded, blue curve is for average and the green curve is for the minimum power recorded during the measurement.
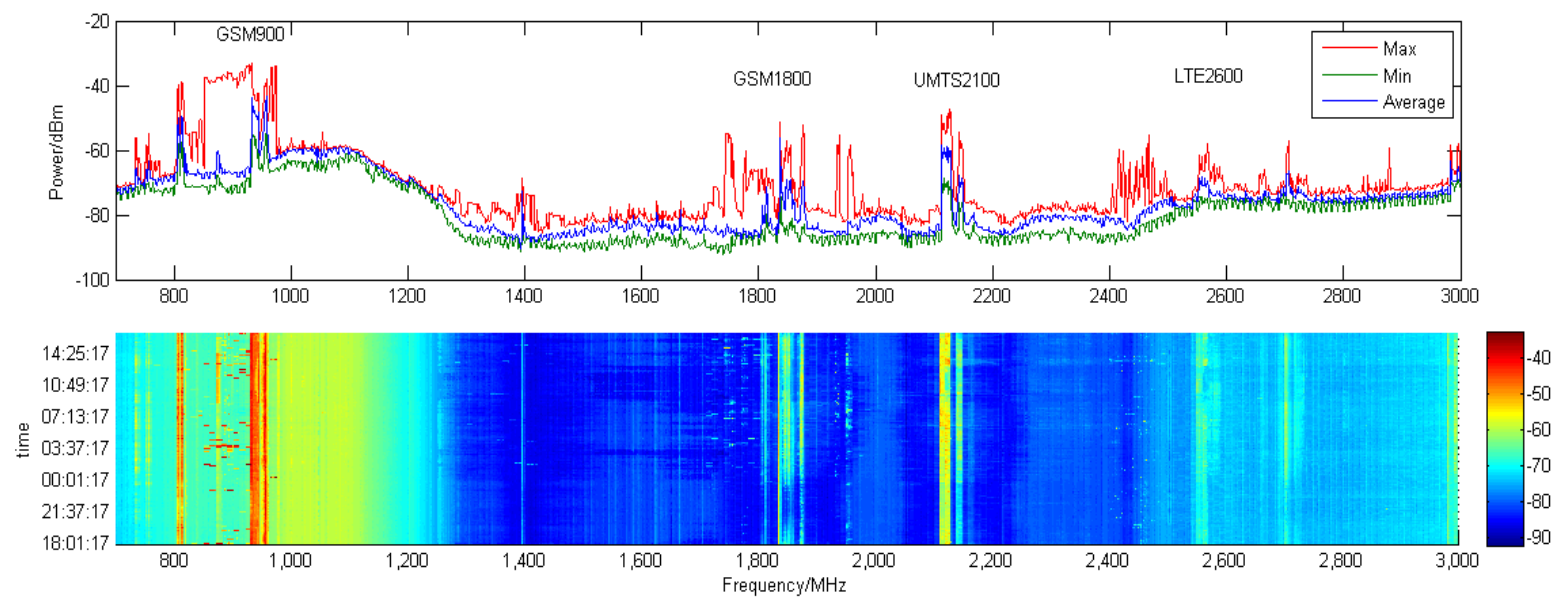

Figure 3: Measured Power versus frequency (700MHz-3000MHz) for 24 hours

\subsection{The Telecommunication Frequency Band}

\section{Spectrum Occupancy}

The occupancy for the telecommunication bands GSM900, GSM1800, UMTS2100 and LTE2600 has been considered. It should however be noted that the occupancy of the telecomunication uplink and downlink in the analysed bands is not identical. The control channels for telecommunication technologies are constantly being broadcasted by the base stations on the downlink (DL), these frequencies seems fully occupied as they are always transmitting with relatively high power. The uplink, on the other hand, for cellular systems, is based on active user.

\subsubsection{GSM900 Frequency Band}

The first telecommunication frequency band considered in the study was the GSM900. Figure 4 and figure 5 show the occupancy for the GSM900 uplink and Downlink respectively. These bands have an average occupancy/duty cycle obtained from the figure of $8.8 \%$ and $52.4 \%$ respectively. This band has the highest utilization level experienced.
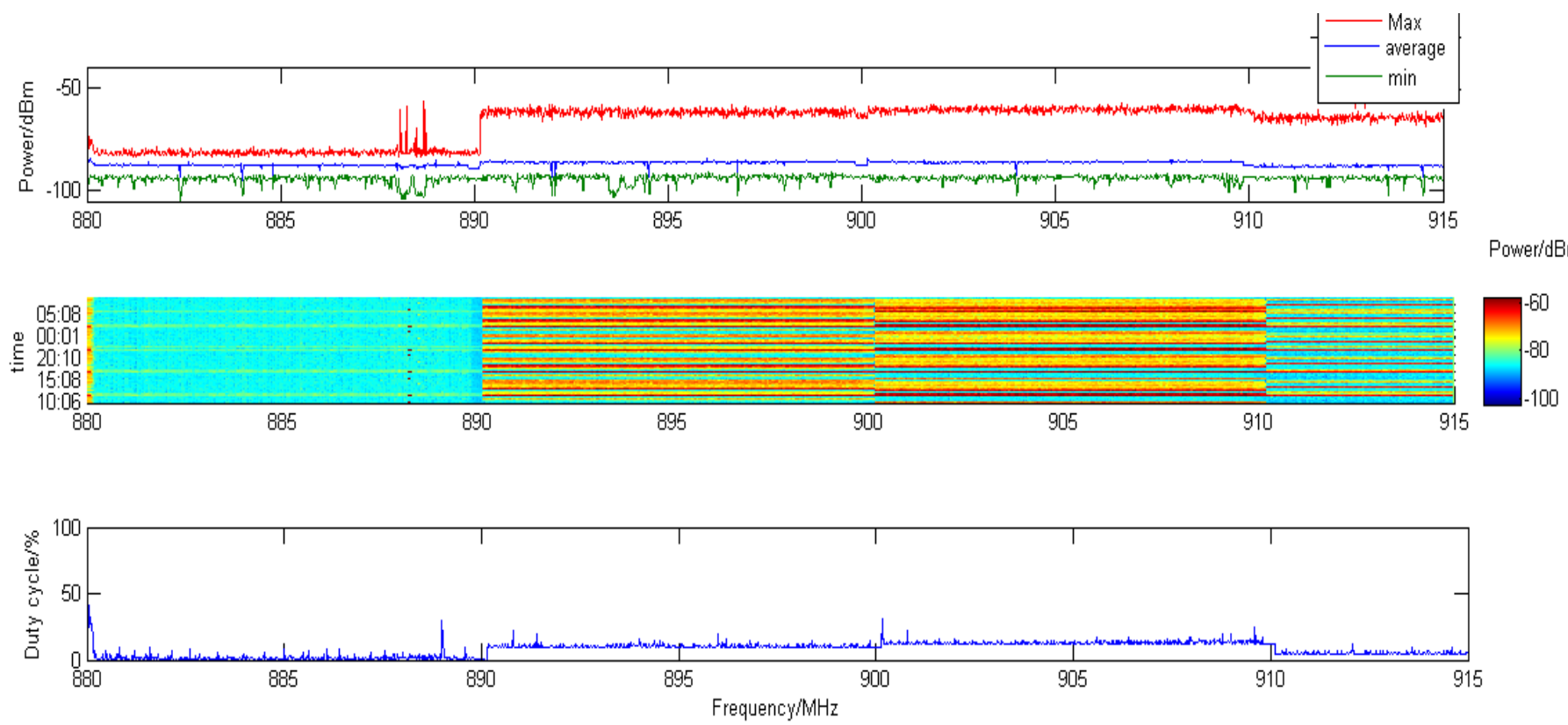

Figure 4: GSM900 Uplink occupancy measurement 

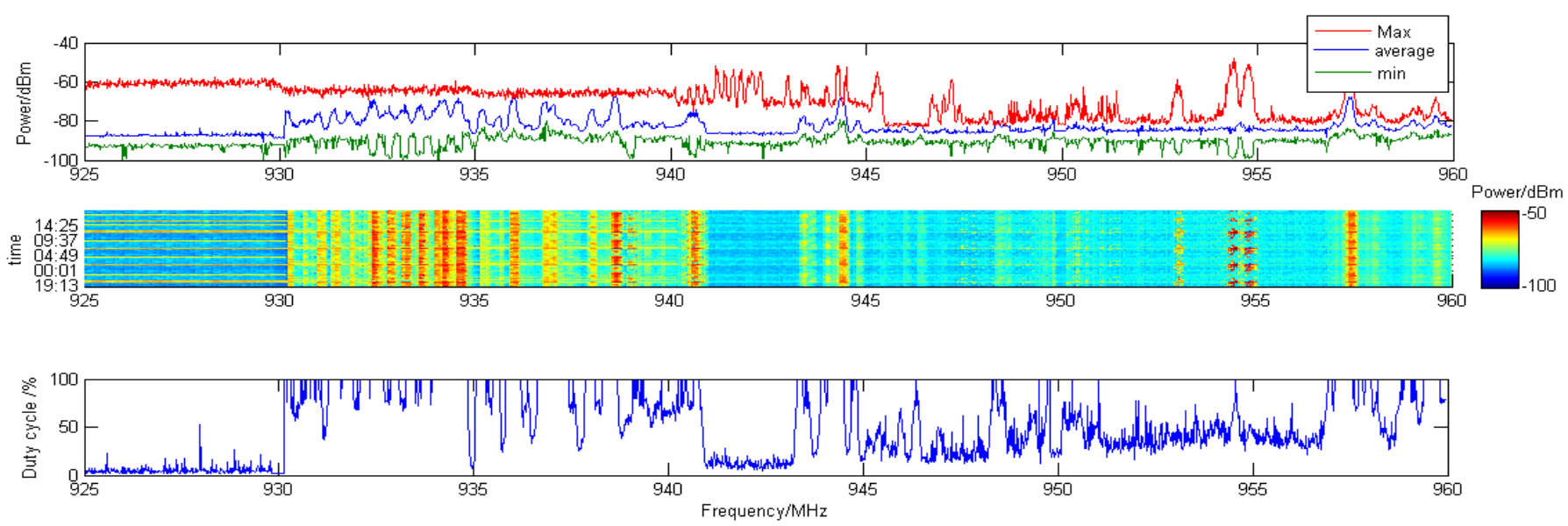

Figure 5: GSM900 Downlink occupancy measurement.

\subsubsection{GSM1800 Frequency Band}

Figure 6 and figure 7 show occupancy in the GSM1800 telecommunication band. The band shows occupancy of $0.6 \%$ and $13.6 \%$.
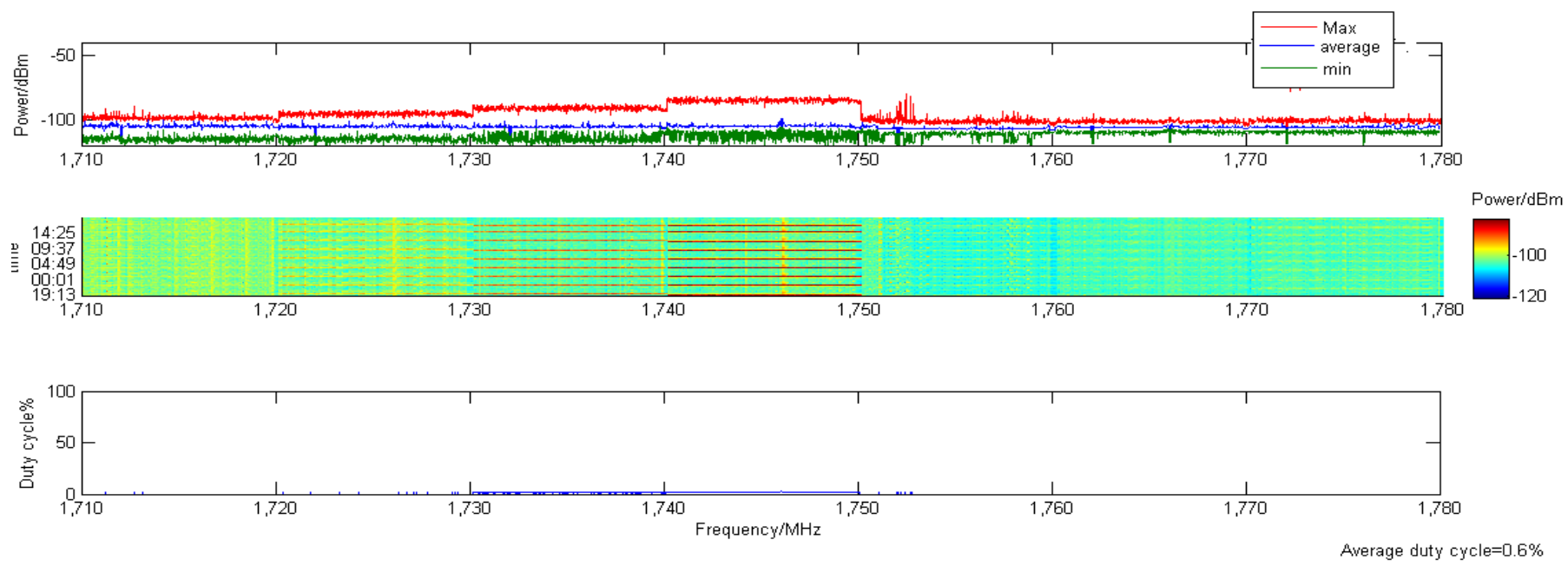

Figure 6: GSM1800 Uplink occupancy measurement
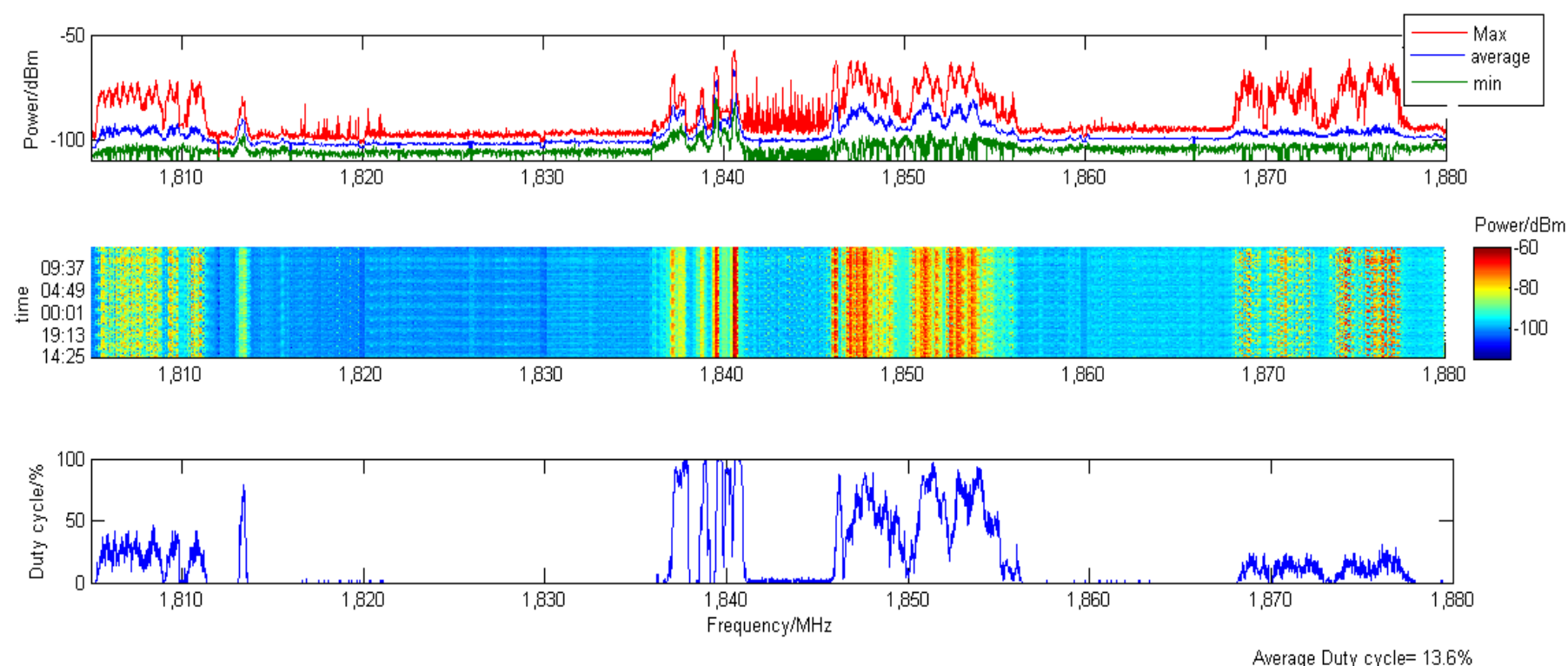


\subsubsection{UMTS2100 Frequency Band}

Figure 7: GSM1800 Downlink occupancy measurement

This band is for $3 \mathrm{G}$ comunication Figure 8 and figure 9 show measured ocupancy in this band.
The average occupancy for the uplink is $0.56 \%$ and for the downlink the ocupancy $48.7 \%$
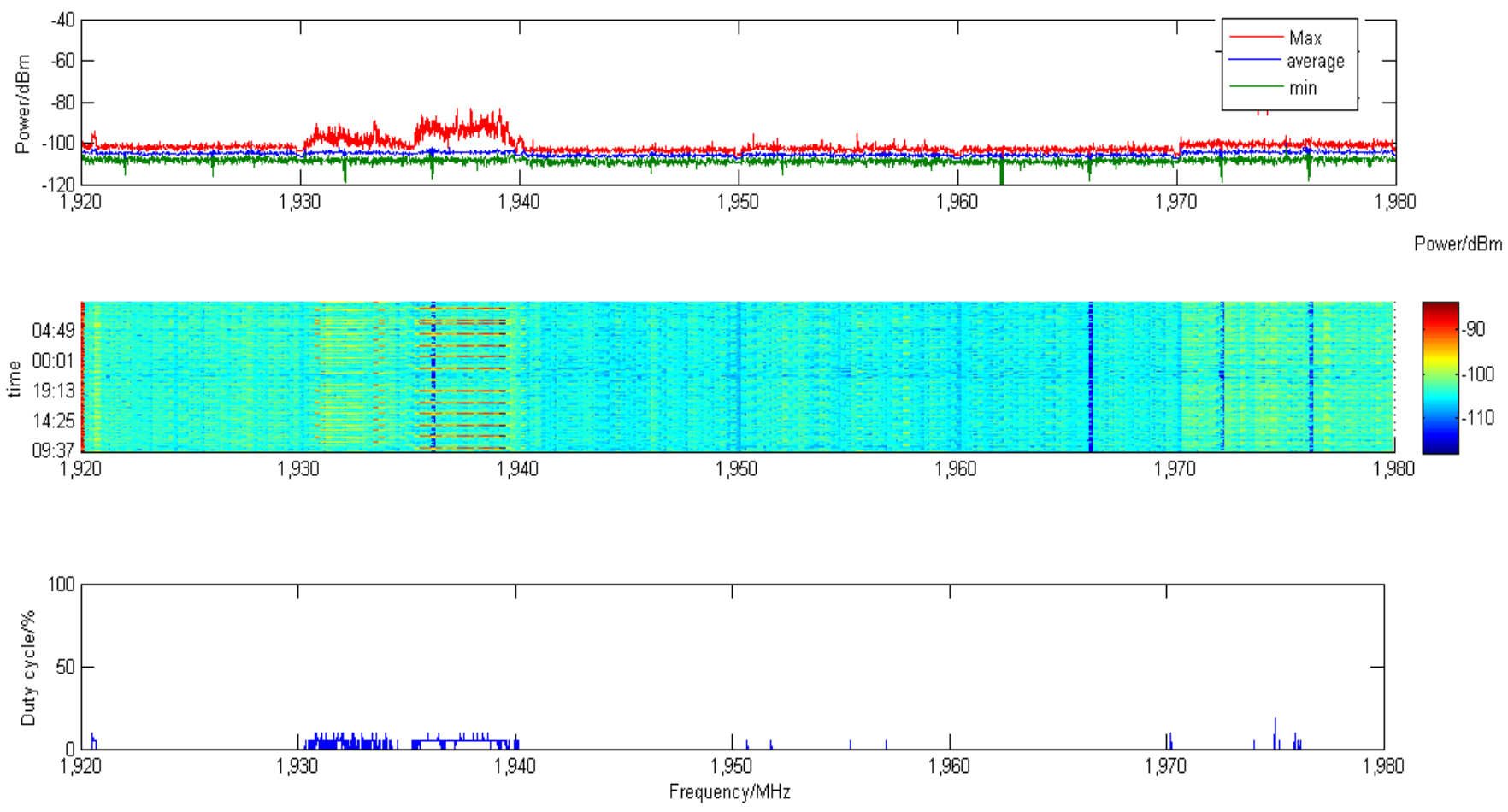

Average Duty cycle $=0.56 \%$

Figure 8: UMTS2100 Uplink occupancy measurement
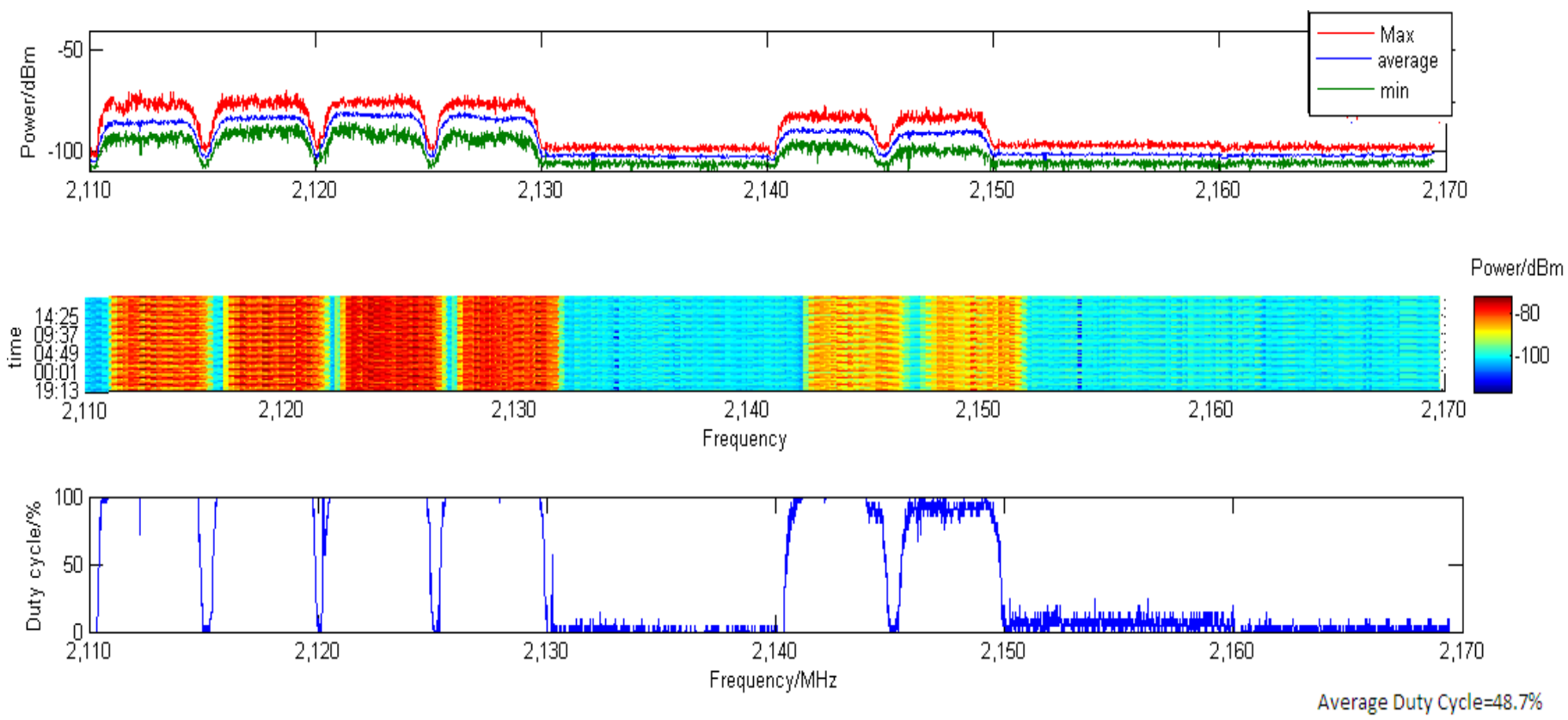

Figure 9: UMTS2100 Downlink occupancy measurement

\subsubsection{LTE2600 Frequency band}

The LTE band is for $4 \mathrm{G}$ technology. At the point of measurement this band showed approximately $0 \%$ occupancy. Figure 10 and figure 11 show the measure occupancy in the uplink and downlink 

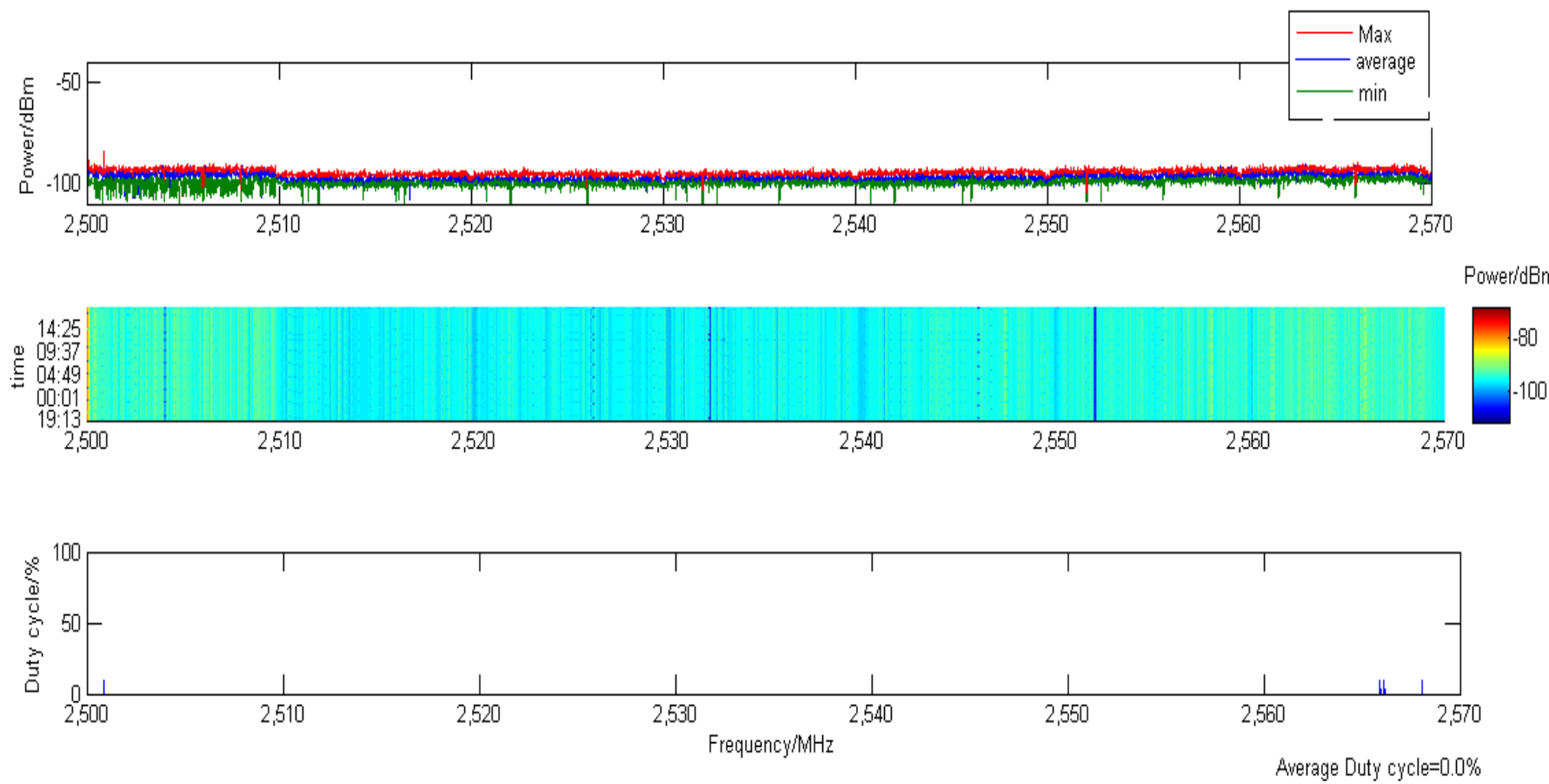

Figure 10: LTE2600 Uplink occupancy measurement

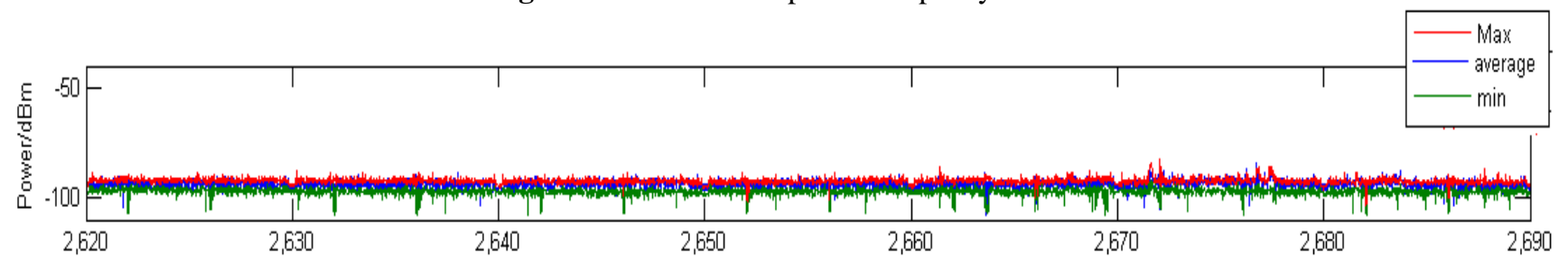

PoweridBm
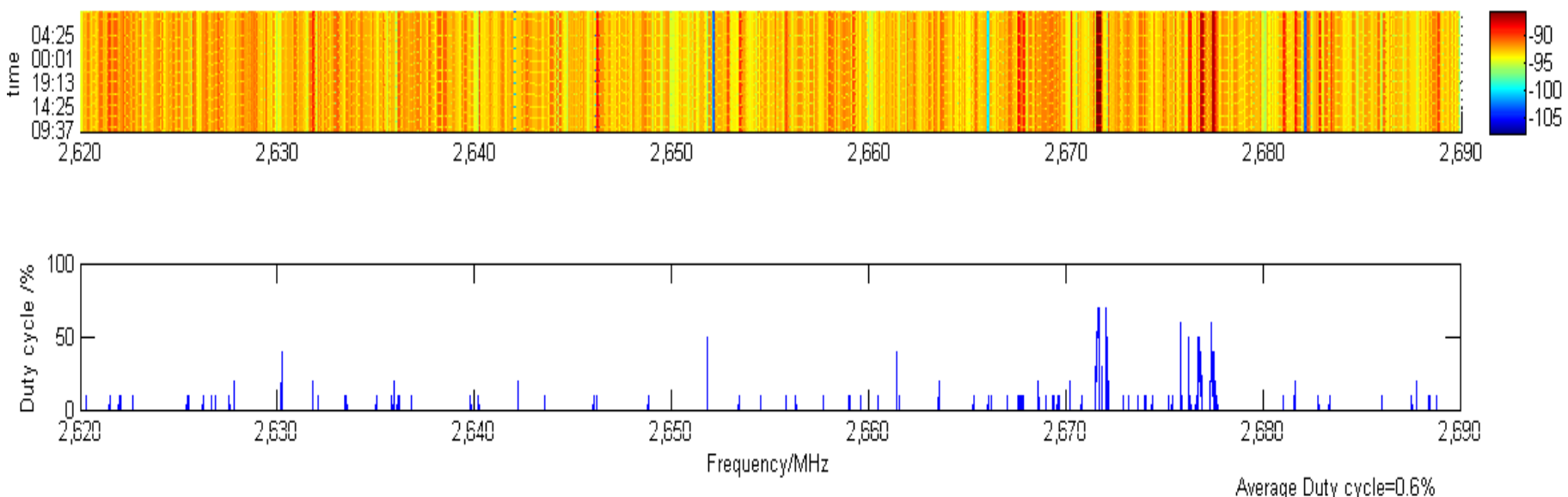

Figure 11: LTE2600 Downlink occupancy measurement

\section{CONCLUSION}

In this paper spectrum Utilization of telecommunication bands in Kampala has been measured and analysed. The measurements show that the highly utilized spectrum is the GSM900 and the LTE2600 is the most underutilized. The result indicates abundant potential for cognitive radio deployment in all the telecommunication bands. It should however be noted that the utilization level of a particular band cannot be extended to other bands or other locations.

\section{REFERENCES}

[1]. Meftah Mehdawi, N. Riley, K. Paulson, A. Fanan and M. Ammar. Spectrum Occupancy Survey In HULL-UK For Cognitive Radio Applications: Measurement \& Analysis, international journal of scientific \& technology research, vol 2, issue 4, 2013

[2]. Sanders, F. H. Broadband spectrum surveys in Denver, CO, San Diego, CA, and Los Angeles, CA: 
methodology, analysis, and comparative results, in Proc. 1998 IEEE International Symposium on Electromagnetic Compatibility, 1998, vol. 2, pp. 988-993.

[3]. Biggs, M. Henley, A. Clarkson, T. Occupancy analysis of the $2.4 \mathrm{GHz}$ ISM band, IEEE Proc. on Comms, 2004, vol. 151, pp. 481-488

[4]. Barnes S.D, Jansen van Vuuren P.A and Maharaj B.T. Spectrum occupancy investigation: Measurements in South Africa. Measurement 46, 2013, pp 3098-3112.

[5]. Bara'u Gafai Najashi , Feng Wenjiang and Choiabu Kadri. An Insight into Spectrum Occupancy in Nigeria, International Journal of Computer Science, Vol. 10, Issue 1, 2013, pp 394-399.

[6]. Jiantao Xue, Zhiyong Feng and Ping Zhang. Spectrum Occupancy Measurements and Analysis in Beijing. International Conference on Electronic Engineering and Computer Science, Vol4, 2013, pp295-302

[7]. Alexandru Marţian, Călin Vlădeanu, Ioana Marcu and Ion Marghescu. Evaluation of Spectrum Occupancy in an Urban Environment in a Cognitive Radio Context. International Journal on Advances in Telecommunications, vol 3, 2010, pp 172-181.

[8]. Mark A. McHenry, Dan McCloskey, Dennis Roberson and John T. MacDonald, Spectrum Occupancy Measurements Chicago, Illinois, Wireless Interference: Characterization and Impact on Network Performance project, 2005

[9]. Salim A. Hanna et. al, "Spectrum metrics for $2.4 \mathrm{GHz}$ ISM Band Cognitive Radio Applications", IEEE $22^{\text {nd }}$ International Symposium on Personal, Indoor, and Mobile Radio Communications. 2011

[10]. Yanfeng Han et al. "Spectrum Occupancy Measurement: Focus on the TV Frequency",International Conference on Signal Processing System, 2010.

[11]. Oliver Holland et. al, "Spectrum Power Measurements in 2G and 3G Cellular Phone Bands during the 2006 Football World Cup in Germany", New Frontiers in Dynamic Spectrum Access Networks, 2007. 